\title{
Esquizofrenia: o que analistas do comportamento têm feito no Brasil?
}

\section{Schizophrenia: what behavior analysts have done in Brazil?}

\section{Esquizofrenia: qué han estado haciendo los analistas del comportamiento en Brasil?}

\section{Carlos Henrique Santos da Silva ${ }^{1}$}

[1] Pontifícia Universidade Católica de São Paulo | Título abreviado: Esquizofrenia: o que analistas do comportamento têm feito no Brasil? | Endereço para correspondência: Endereço: Rua Bartira, 387. Perdizes. São Paulo-SP. CEP: 05009-000 | Email: rriques@gmail.coml doi: 10.18761/PAC.2020.v11.n2.02 
Abstract: Studies conducted out of Brazil since 1960's indicated the effectiveness of Behavior Analysis procedures in individuals with schizophrenia diagnosis. The objectives, target-behaviors, procedures and reinforcers used were several. The present research attempted to realize a review in Brazilian literature including studies conducted with schizophrenics, which used Behavior Analysis procedures. Nine database were selected and a set of key words identified 19 Brazilian studies, which showed constant publications, mostly of dissertations and thesis, since 2005. Unappropriated and appropriated vocal verbal behavior showed up in most interventions with both differential reinforcement of alternative behaviors and extinction procedures. A small amount of experiments selected as target behavior aggression, personal hygiene, social abilities, reading, and also revealed effectiveness, suggesting the lack of more studies that comprise other target behaviors.

Keywords: Schizophrenia, Behavior Analysis, Psychotic behavior.

Resumen: Los estudios realizados en el extranjero desde la década de 1960 apuntan a la efectividad de los procedimientos de análisis de comportamiento en personas diagnosticadas con esquizofrenia. Hubo muchos objetivos, comportamientos seleccionados, procedimientos y refuerzos utilizados. El presente estudio tuvo como objetivo realizar una revisión de la literatura brasileña sobre la investigación realizada con personas con esquizofrenia en un enfoque analítico-conductual. Se seleccionaron nueve bases de datos y un conjunto de palabras clave identificó 19 estudios nacionales, que señalaron publicaciones constantes, principalmente de tesis y disertaciones, sobre el tema desde 2005. El comportamiento verbal apropiado e inapropiado apareció con mayor prominencia en las intervenciones realizadas bajo los procedimientos de refuerzo diferencial para respuestas alternativas y extinción. Pocos estudios han dirigido respuestas a la agresión, la higiene personal, las habilidades sociales, la lectura y también han demostrado su efectividad, lo que sugiere la necesidad de realizar más estudios que abarquen otros comportamientos.

Palabras clave: Esquizofrenia; Análisis del comportamiento; Comportamiento psicótico. 
A esquizofrenia, no Manual Diagnóstico e Estatístico de Transtornos Mentais DMS-V (APA, 2013/2014), está inclusa no Espectro da Esquizofrenia e Outros Transtornos Psicóticos, os quais são definidos por prejuízos em pelo menos um dos seguintes domínios: delírios, alucinações, pensamento (discurso) desorganizado, comportamento motor grosseiramente desorganizado ou anormal (incluindo catatonia) e apresentação de sintomas negativos (e.g., expressão emocional diminuída, avolia, alogia, anedonia e falta de sociabilidade).

Embora o modelo médico de diagnóstico proposto pelo DSM inclua os indivíduos que apresentam determinados tipos de comportamentos em classificações com base em critérios diagnósticos, o sistema representa apenas uma descrição de topografias e frequências de respostas que são apresentadas pelos indivíduos (Banaco, 1999). Respostas verbais, consideradas bizarras, são psicopatologizadas como sintoma da esquizofrenia e a busca de explicações para esses comportamentos acarretam em pressupostos simplistas, com argumentos de ordem mental ou fisiológica (Britto, 2005).

Skinner (1959) ao definir o comportamento do esquizofrênico afirmou que: "o comportamento psicótico, como qualquer comportamento, é parte do mundo de eventos observáveis, aos quais os métodos da ciência natural são aplicáveis" (p. 219). Laying e Andronis (1984) discutem que o comportamento alucinatório do esquizofrênico é comportamento operante, cuja frequência é mantida pelas contingências de reforçamento. As consequências que mantém essas respostas podem ser múltiplas, envolvendo reforçamento positivo ou negativo e suas relações com as respostas podem ser complexas. Os estímulos que antecedem o responder podem ser inacessíveis à audiência, como é o caso de privação e de estimulação aversiva.

Alucinações e delírios são defendidos por Britto $(2004,2005)$ como comportamentos verbais. É necessária a realização de avaliação funcional, incluindo observação e manipulação de variáveis, para a compreensão dos motivos que contribuem para a ocorrência desses comportamentos-problema.

As primeiras pesquisas realizadas com esquizofrênicos foram conduzidas na década de 50, por Skinner, Solomon e Lindsley em instituições psiquiátricas. Todavia, os experimentos não foram conduzidos na tentativa de modificação de comportamentos-problema em situação natural, mas sim para mostrar que os princípios operantes eram aplicáveis também em humanos. No início dos anos 60 pesquisas sobre comportamento verbal foram adquirindo relevância e, desta forma, experimentos com esquizofrênicos foram realizados com o objetivo de diminuir a frequência de respostas verbais inadequadas e aumentar as que eram consideradas adequadas por meio dos procedimentos de reforçamento e extinção (Kazdin, 1978).

Um dos estudos realizados foi o de Ayllon e Haughton (1964), no qual uma paciente diagnosticada com esquizofrenia crônica apresentava repostas verbais delirantes há 14 anos. Duas classes de respostas passaram por intervenção: respostas verbais psicóticas e as respostas verbais neutras (não psicóticas). Os procedimentos de reforçamento (e.g., prestar atenção, conversar, oferecer cigarros e pedaços de doces) e extinção (e.g., parecer ocupado, não olhar para a paciente) foram aplicados às duas classes de respostas após a linha de base. Os resultados mostraram que a frequência de respostas psicóticas e neutras, quando consequenciadas com atenção social, aumentava até o dobro do número registrado na linha de base e houve um decréscimo considerável quando o procedimento de extinção entrou em vigor. Os achados experimentais levaram os autores a discutir que a frequência de diferentes classes de respostas verbais é dependente de contingências de reforçamento do ambiente social.

Estudos conduzidos posteriormente (DeLeon, Arnold, Rodriguez-Catter \& Uy, 2003; Dixon, Benedict \& Larson, 2001; Liberman, Teigen, Patterson \& Baker, 1973; Mace \& Lalli, 1991; Wilder, Masuda, O'Connor \& Baham, 2001) também apontaram que o que é chamado de fala psicótica ou fala inapropriada é mantido por atenção social contingente à resposta. Ainda, mostraram que os procedimentos de extinção e reforçamento ou reforçamento de respostas alternativas (DRA) pareceram efetivos para a diminuição nas falas inapropriadas e aumento nas apropriadas.

Outra técnica que foi aplicada em pessoas com o diagnóstico de esquizofrenia foi a economia de fichas (token economy), que foi um marco na Análise Aplicada do Comportamento para diversas populações (Kazdin, 1978). Dentre os estudos, destaca- 
-se o de Ayllon e Azrin (1965), no qual pacientes de um hospital psiquiátrico recebiam fichas por se engajarem em atividades, o que os autores chamaram de comportamentos adaptativos, e poderiam posteriormente ser trocados por uma variedade de reforçadores. Os resultados mostraram que o reforçamento por fichas de forma contingente é uma técnica eficaz em aumentar a frequência de respostas adaptativas em esquizofrênicos.

Dentre os comportamentos-alvo que foram foco dos estudos com economia de fichas estavam: comportamento agressivo, berros e apatia (Winkler, 1970); falas bizarras (Wincze, Leitenberg \& Agras, 1972); falar com pessoas, perguntar e responder questões e trabalhar de forma cooperativa (Bennett \& Maley, 1973); fazer afirmações positivas sobre pessoas e atividades (Tracey, Briddell \& Wilson, 1974), higiene pessoal, trabalho, autogerenciamento e habilidades sociais (Nelson \& Cone, 1979).

Algumas dessas intervenções mostraram ser efetivas, mas não em todas as aplicações (Glynn, 1990). Em alguns casos, o uso de reforçadores arbitrários foi manipulado sem a análise funcional das variáveis que controlavam o comportamento-alvo, procedimento necessário para explicar o problema no modelo operante e, por conseguinte, realizar a intervenção (Schock, Clay \& Cipani, 1998).

Uma metodologia foi desenvolvida por Iwata, Dorsey, Slifer, Bauman e Richman (1994) para modificar comportamentos autolesivos de pessoas com problemas de desenvolvimento. Os autores propuseram que, antes da intervenção, seja realizada avaliação de forma indireta (e.g., questionários, entrevistas), observação do comportamento-problema no ambiente natural e, por fim, o que foi chamado de análise funcional experimental, manipulando cada variável que favorece a emissão da resposta. Após a proposta de Iwata et al. (1994), estudos com esquizofrênicos realizaram avaliação indireta, observações do comportamento-problema e análise funcional experimental antes da intervenção para demonstração das variáveis de controle (DeLeon et al., 2003; Dixon et al., 2001; Wilder et al., 2001).

Desde o início da aplicação dos princípios comportamentais em humanos parece que houve uma preocupação na demonstração de tais princípios e a elaboração de intervenções para pessoas com o diagnóstico de esquizofrenia. Além de artigos teóricos-conceituais e experimentais, ressaltam-se também estudos de revisão da literatura, como o de Scotti, McMorrow e Trawitzki (1993). Os autores realizaram a revisão por meio de nove principais jornais e abrangendo o período de 1963 até 1988. Os resultados apontaram aumento substancial na média de publicações em esquizofrenia por ano na década de 70, em relação às décadas de 60 e 80 . Os procedimentos mais utilizados foram os baseados em reforçamento (e.g., modelagem, modelação), Contingency Management (e.g., economia de fichas, contingências de grupo) e punição.

Um levantamento parcial da literatura foi realizado por Martone e Zamignani (2002), incluindo a década de 90, não abarcada no estudo de Scotti et al. (1993), e envolvendo três revistas de Análise do Comportamento: Journal of The Experimental Analysis of Behavior, Journal of Applied Behavior Analysis e Behavior Research and Therapy. Os autores encontraram um grande número de publicações nas décadas de 60 e 70, seguido de um decréscimo nos anos de 80 e 90 e concluíram que não houve progresso no desenvolvimento de intervenções mais abrangentes. A ênfase tem sido na mudança de respostas discretas.

A diminuição nas publicações após o ápice inicial, nos anos 60 e 70, talvez pode ser explicada pelo fato de haver uma relação direta entre crescimento de intervenções na área e críticas no que dizia respeito à: limitação no alcance de técnicas utilizadas, utilização de procedimentos aversivos e dificuldades em mostrar generalização dos resultados obtidos, o que pode estar relacionado com a limitação do setting (instituições) (Guedes, 1993; Holland, 1978).

Outra revisão (Santos, Santos \& Aureliano, 2013) levantou pesquisas publicadas no Journal of The Experimental Analysis of Behavior e no Journal of Applied Behavior Analysis entre 1959 e 2011, contemplando o período ainda não analisado em outros estudos. Um total de 25 artigos foi selecionado pelas autoras, que indicaram um aumento de publicações entre as décadas de 60 e 70, seguido de uma diminuição nas décadas de 80, 90 e 2000. O principal objetivo dos artigos foram: avaliar a efetividade dos princípios operantes (anos 60 e 80) e modificar o comportamento do participante (anos 70, 90 e 2000). Dentre os comportamentos-alvo que passaram por intervenção, destacam-se: alimentação e comporta- 
mento verbal na década de 60 , comportamento verbal nos anos 70, 80 e 2000 e atividades domésticas nos anos 90. As autoras tiveram outras classificações para comportamentos-alvo, dentre elas: autocuidado, habilidades sociais, comportamentos agressivos e outros excessos comportamentais. Todavia, tal variedade de variáveis dependentes aparecem apenas nas duas primeiras décadas (60 e 70). Nas posteriores (80, 90 e 2000) aparecem apenas: comportamento verbal, habilidades sociais e comportamentos agressivos (Santos et al., 2013).

Em relação aos reforçadores utilizados nas pesquisas, notou-se uma variedade de estímulos utilizados nos anos 60 e 70. Na década de 60 destaca-se a utilização de reforçadores condicionados e generalizados, seguido por reforçadores sociais e primários. Na década de 70 os reforçadores generalizados continuaram entre os mais utilizados, seguidos por reforçadores sociais. Já nas décadas seguintes houve diminuição na variedade de reforçadores. Nos anos 80, apenas reforçadores generalizados foram empregados. Nas décadas de 90 e 2000, os reforçadores sociais foram os mais utilizados (Santos et al., 2013).

O estudo de Santos et al. (2013) apontou ainda que, embora haja um decréscimo nas publicações sobre esquizofrenia na Análise do Comportamento nas últimas cinco décadas na literatura estrangeira e nas revistas selecionadas, houve aumento na produção de teses e dissertações sobre o tema no Brasil. Levando em consideração que as revisões realizadas abarcam quase todo o período de existência dos principais jornais estrangeiros (Martone \& Zamignani, 2002; Santos et al., 2013; Scotti et al., 1993) e também o fato de que nenhuma delas incluiu em suas buscas os periódicos brasileiros, torna-se importante realizar um levantamento de pesquisas no Brasil.

O objetivo deste estudo, portanto, foi realizar uma revisão da literatura brasileira sobre esquizofrenia no referencial da Análise do Comportamento. Pretendeu-se ainda: (1) incluir teses e dissertações, além de artigos publicados em periódicos nacionais e (2) analisar que tipos de intervenções têm sido realizadas e os principais resultados obtidos. Conhecer o que tem sido feito no Brasil é importante para que sejam identificadas intervenções que se mostraram eficazes para profissionais que atuam com pessoas com o diagnóstico de esquizofrenia. Ainda, pode elucidar novas pesquisas que manipulem variáveis ainda não investigadas ou mesmo variáveis que carecem de compreensão.

\section{Método}

\section{Materiais}

Artigos que abordassem a esquizofrenia sob a ótica da Análise do Comportamento foram utilizados. Os estudos foram acessados por meio de nove bases de dados: Capes, Scielo, Pepsic, Biblioteca Virtual em Saúde, Psycinfo, Web of Science, Scopus, Pubmed e Biblioteca Digital de Teses e Dissertações.

\section{Procedimento}

O site http://www.periodicos.capes.gov.br foi acessado e cada base de dados foi encontrada por meio da opção "buscar base". Dentro de cada base as buscas foram realizadas pela opção "busca avançada". As seguintes palavras-chave foram utilizadas: "esquizofrenia", "esquizofrênico (a)", "psicótico (a)" e "psicose" combinadas com "análise do comportamento", "comportamental", "behaviorismo", "análise funcional" e "comportamento". Todas as buscas foram realizadas escolhendo, para cada palavra, a opção "em qualquer campo" e em todos os anos.

Os títulos e resumos dos estudos resultantes das buscas foram lidos e os seguintes critérios de seleção foram aplicados: (1) estudos sob o referencial da Análise do Comportamento e (2) estudos experimentais de pesquisa básica e aplicada , cujos participantes possuíam diagnóstico de esquizofrenia. O critério de exclusão empregado abarcou estudos que não foram realizados no Brasil. Quando não foi possível aplicar os critérios de seleção por meio da leitura dos resumos, optou-se pela leitura do método do estudo.

Uma planilha foi criada no Microsoft Excel com as categorias de análise, a citar: autor, ano, revista publicada, filiação, setting, objetivo, delineamento experimental utilizado, realização de avaliação funcional experimental, variável dependente, variável independente, reforçadores utilizados, principais resultados da intervenção, follow-up. 
Concordância entre observadores

Um observador treinado categorizou 20\% dos estudos selecionados. O cálculo do índice de concordância foi efetuado utilizando a fórmula padrão: [Concordância/(Discordância + Concordância)] $\mathrm{x} 100$. O percentual de fidedignidade foi calculado para cada categoria e o resultado alcançou índices que variaram de 91 a $98 \%$.

\section{Resultados e Discussão}

As buscas resultaram um total de 808 estudos. Após a aplicação dos critérios de inclusão e exclusão com a eliminação de repetidos restaram 19. Importante ressaltar que, além desses, foram encontrados cinco artigos teóricos e de revisão por meio das buscas, mas não foram incluídos na presente análise.

Quatro artigos foram publicados nos periódicos Revista Brasileira de Terapia Comportamental e Cognitiva, Revista Brasileira de Análise do Comportamento e Psicologia: Teoria e Pesquisa. O restante (15) refere-se à dissertação ou tese encontrados na Biblioteca Digital de Teses e Dissertações, sendo 12 dissertações de mestrado e três teses de doutorado. Em relação à filiação dos autores, quase todos os estudos são provenientes da Pontifícia Universidade Católica de Goiás, com exceção de uma dissertação que foi realizada na Pontifícia Universidade Católica de São Paulo.
A Figura 1 mostra a distribuição dos estudos no decorrer dos anos. Notou-se que desde 2005 até 2018 houve estudos sobre esquizofrenia na Análise do Comportamento no Brasil, com exceção do ano de 2017. Os anos de 2005 e 2010 foram os que mais tiveram publicações, três estudos em cada, seguidos pelos anos de 2012 e 2014 com dois estudos cada.

Estes dados confirmam a observação de Santos et al. (2013) de que, no Brasil, houve aumento no número de publicações de teses e dissertações nos últimos anos, em contraste com a diminuição no número de publicações sobre o tema no exterior (Martone \& Zamignani, 2002; Santos et al., 2013).

Os comportamentos-alvo selecionados e os tipos de reforçadores utilizados nos estudos são mostrados na Figura 2. Um mesmo estudo pode ter sido contabilizado mais de uma vez, visto que alguns trabalharam com mais de uma variável dependente. Em relação às respostas-alvo, na maioria das publicações (16), o comportamento verbal dos participantes foi foco de intervenções. Destes, 13 estiveram relacionados com falas apropriadas e inapropriadas. Dentre os três restantes, um trabalhou com a classe de respostas de falar sobre si e a instalação de mandos. Um teve como foco o volume baixo da voz e verbalizações curtas e um trabalhou com leitura, conversas e falas inadequadas.

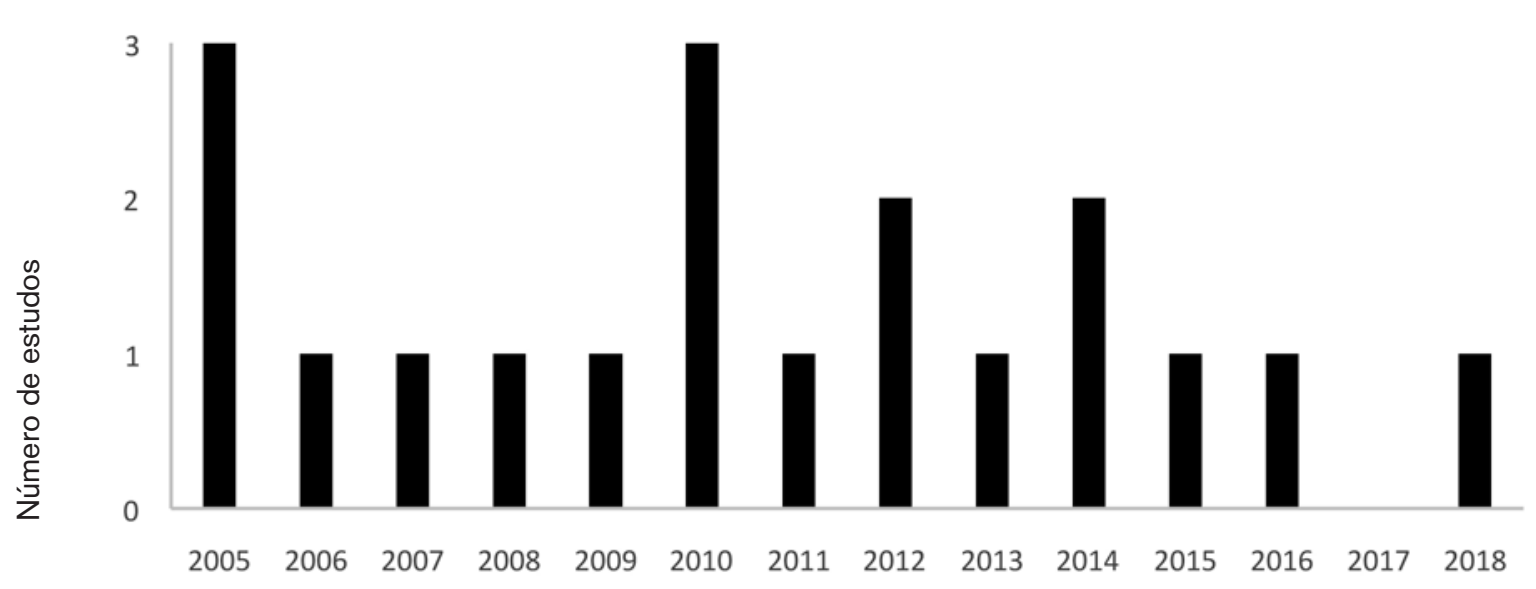

Anos

Figura 1. Número de publicações por ano 
Três estudos tiveram como alvo respostas de autocuidado (e.g., beber água no copo, lavar as mãos, escovar os dentes) e outros três tiveram como foco comportamentos agressivos (e.g., agarrar ou unhar o braço de outras pessoas, dar tapas, ameaçar, empurrar). Habilidades sociais aparecem em quatro estudos. Outros comportamentos-alvo (e.g., executar tarefas solicitadas, desenhar, ficar sozinho sem ter atenção, recusa na participação de tarefas, bater palmas em atividade grupal e executar atividades no tempo livre) foram encontrados em três publicações.

A variedade de comportamentos-alvo encontrada nos artigos em anos iniciais de publicação no JEAB (Santos et al., 2013) parece se aplicar aos dois primeiros anos de publicações dos estudos brasileiros. Nos anos seguintes a maioria dos estudos teve seu foco concentrado na diminuição na frequência de classes de respostas verbais vocais inapropriadas e o aumento na frequência de classes de respostas verbais vocais consideradas apropriadas. Desde 2013, os estudos tiveram como alvo estas duas clas- ses de respostas. As demais aparecem nas publicações entre 2005 e 2012, concentradas em maior variedade entre 2005 e 2006. O comportamento verbal foi foco de intervenção tanto no Brasil nos últimos oito anos quanto no exterior na década de 2000.

Os estímulos que foram mais utilizados nas intervenções foram os reforçadores sociais, aparecendo em 12 estudos, seguidos de seis estudos que utilizaram reforçadores sociais em conjunto com reforçadores primários. Santos et al. (2013) apontaram que nas publicações estrangeiras, nas décadas de 60 e 70, os estímulos mais utilizados foram os reforçadores primários e condicionados. Nas décadas de 90 e 2000 destacam-se o uso de reforçadores sociais seguidos pelos condicionados. Estes dados se contrapõem aos dos estudos brasileiros, que usaram quase que exclusivamente reforçadores sociais de 2005 até 2018 e reforçadores primários em conjunto com os sociais até 2014. Além disso, não foi encontrado outros tipos de reforçadores que poderiam fazer parte das intervenções.

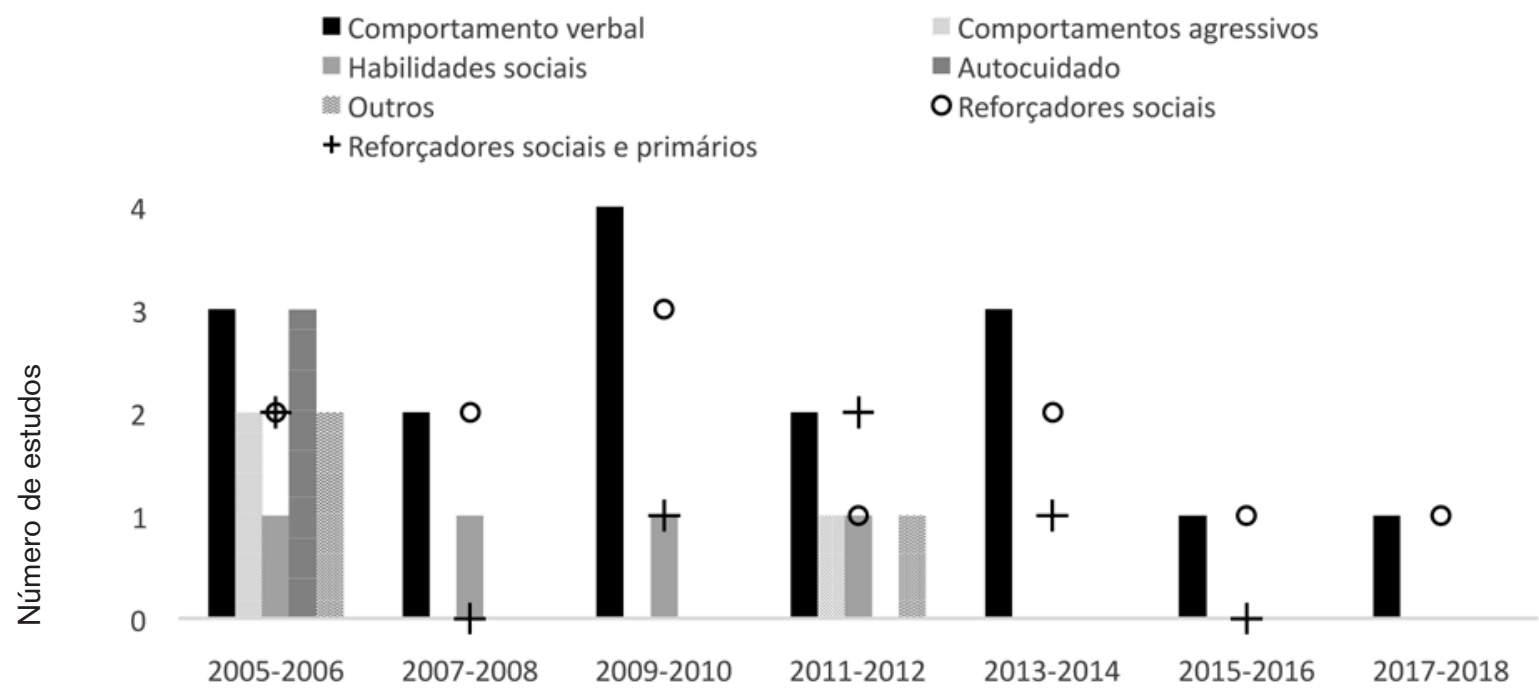

Blocos de dois anos

Figura 2. Respostas alvo (barras) e reforçadores (marcadores) utilizados nos estudos a cada dois anos de publicações. 
Os objetivos dos estudos analisados são mostrados na Figura 3. Estudos com objetivos de demonstrar a efetividade dos procedimentos da Análise do Comportamento em participantes esquizofrênicos (4) e instalar respostas de autocuidado (1) aparecem nos anos iniciais (2005 e 2006) e no ano de 2011. Já entre 2007 a 2018, os estudos objetivaram, em sua maioria, alterar o comportamento verbal do participante (9) ou demonstrar a utilidade da análise funcional experimental (5) proposta por Iwata et al. (1994) ao modificar e adaptar as condições originalmente descritas pelos autores. Nos estudos estrangeiros também há uma predominância de se estudar o comportamento verbal dos participantes com diagnóstico de esquizofrenia desde a década de 60. Além disso, pareceu comum a publicação de estudos em anos iniciais para demonstrar a efetividade dos procedimentos da Análise do Comportamento nos estudos estrangeiros e brasileiros (Santos et al., 2013).

A análise dos estudos selecionados também mostrou que as intervenções, em quatro estudos, foram realizadas apenas no CAPS; sete foram realizadas em instituições psiquiátricas; uma em instituição psiquiátrica e na residência do participante; dois em clínicas; um na clínica, na residência e no templo religioso que o participante frequentava e três apenas nas residências dos participantes. Esses dados sugerem que alguns dos autores têm se preocupado em realizar a intervenção no setting natural do participante ou mesmo em vários ambientes, $o$ que pode facilitar a generalização da intervenção. Além disso, mais da metade dos estudos (10) realizaram follow-up.

Tais características parecem se assemelhar com o escopo proposto pelas pesquisas aplicadas em Análise do Comportamento (Baer, Wolf \& Risley, 1968), cujas principais premissas destacam a realização da pesquisa no ambiente natural no qual o comportamento-alvo possa ser analisado e as variáveis que o controlam ser identificadas e alteradas, visando melhorias no comportamento socialmente relevante. Além disso, Baer et al. (1968) colocam em evidência sete critérios para considerar uma pesquisa como aplicada: aplicada, comportamental, analítica, tecnológica, conceitual, eficaz e generalizável.

Esses critérios revelam a necessidade de a pesquisa ter como objeto de estudo importância para a sociedade (aplicada), possuir a mensuração dos eventos analisados e avaliações explícitas da confiabilidade dos observadores humanos (comportamental), a demonstração da relação (ou não) entre

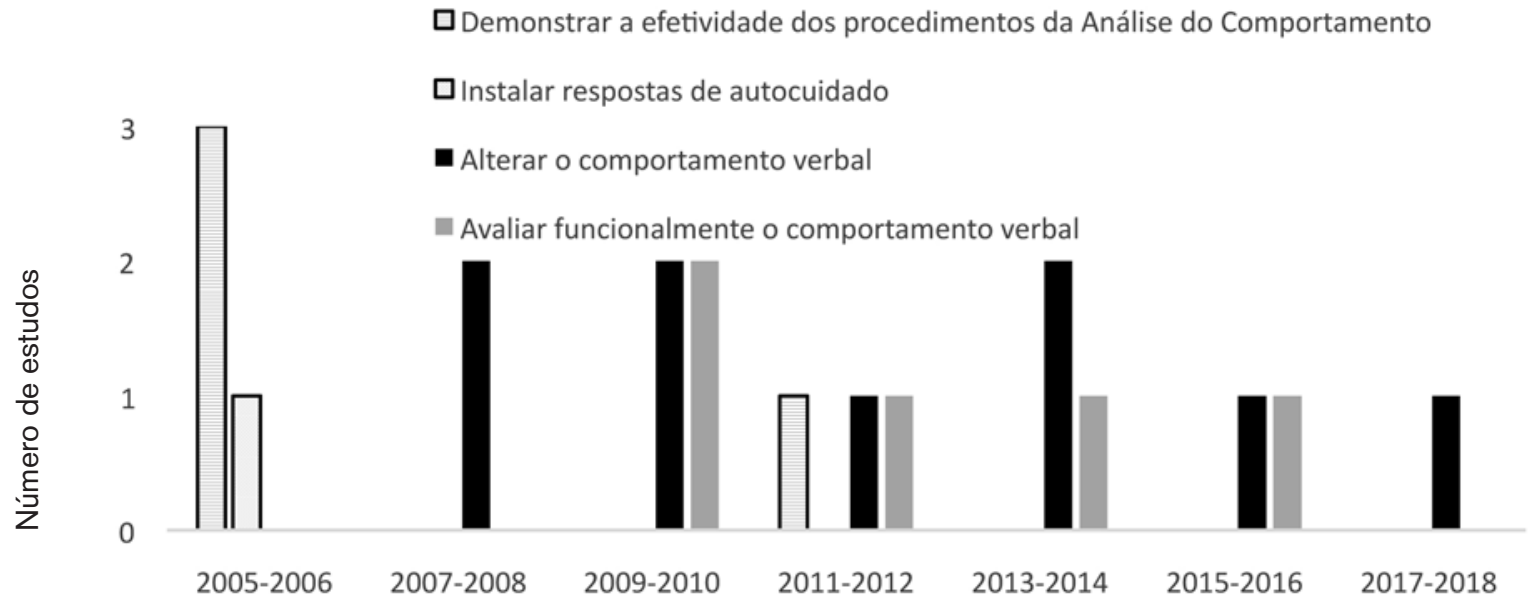

Blocos de dois anos

Figura 3. Principais objetivos dos estudos em blocos de dois anos de publicações. 
eventos ambientais e as classes de respostas (analítica). Ainda, a descrição dos procedimentos de uma pesquisa aplicada deve ser completa, de modo que um leitor treinado consiga replicar e produzir os mesmos resultados (tecnológica), as técnicas empregadas precisam fazer sentido do ponto de vista comportamental (conceitual), produzindo a modificação esperada (eficaz) tanto no ambiente da aplicação quanto em outros ambientes (generalizável).

Em relação à realização da análise funcional experimental antes da intervenção, proposto por Iwata et al. (1994), foi identificada em seis estudos (Alves, 2018; Bueno, 2012; Nogueira, 2016; Rockenbach, 2014; Santana, 2008; Sousa, 2013). Cinco outros estudos (Britto, Rodrigues, Alves \& Quinta, 2010; Marcon, 2010; Marcon, 2014; Marcon \& Britto, 2015; Moura, 2012) foram conduzidos para manipular as condições presentes na análise funcional experimental, mas sem intervenção posterior. Os autores desses estudos preocuparam-se em demonstrar experimentalmente as variáveis das quais o comportamento-alvo é função, antes mesmo de planejar a intervenção apropriada. Essa característica em conjunto com a condução da pesquisa em ambiente natural foram aspectos iden- tificados também em outras pesquisas do programa de Pós-Graduação em Psicologia da Pontifícia Universidade Católica de Goiás, em revisão realizada por Britto e Marcon (2019).

De um modo geral, as análises funcionais dos estudos (Alves, 2018; Britto et al., 2010; Bueno, 2012; Marcon, 2010; Marcon, 2014; Marcon \& Britto, 2015; Moura, 2012; Nogueira, 2016; Rockenbach, 2014; Santana, 2008; Sousa, 2013) mostraram que falas inadequadas podem ser mantidas por atenção social, portanto, por reforçamento positivo demonstrado nas diversas condições de "atenção" ou por reforçamento negativo com a remoção de uma dada atividade contingente com a fala inadequada observado nas condições de demanda. Na condição "controle" os registros indicam menor frequência, quando comparado a atenção ou demanda, sugerindo que a resposta não era controlada por reforçamento automático. Já na condição "sozinho" foi identificado algum registro dessas falas em apenas três pesquisas (Marcon, 2010; Marcon \& Britto, 2015; Nogueira, 2016). Esses dados sugerem que respostas verbais inapropriadas de pessoas com diagnóstico de esquizofrenia podem ser mantidas por contingências de reforçamento (Laying \& Andronis, 1984).

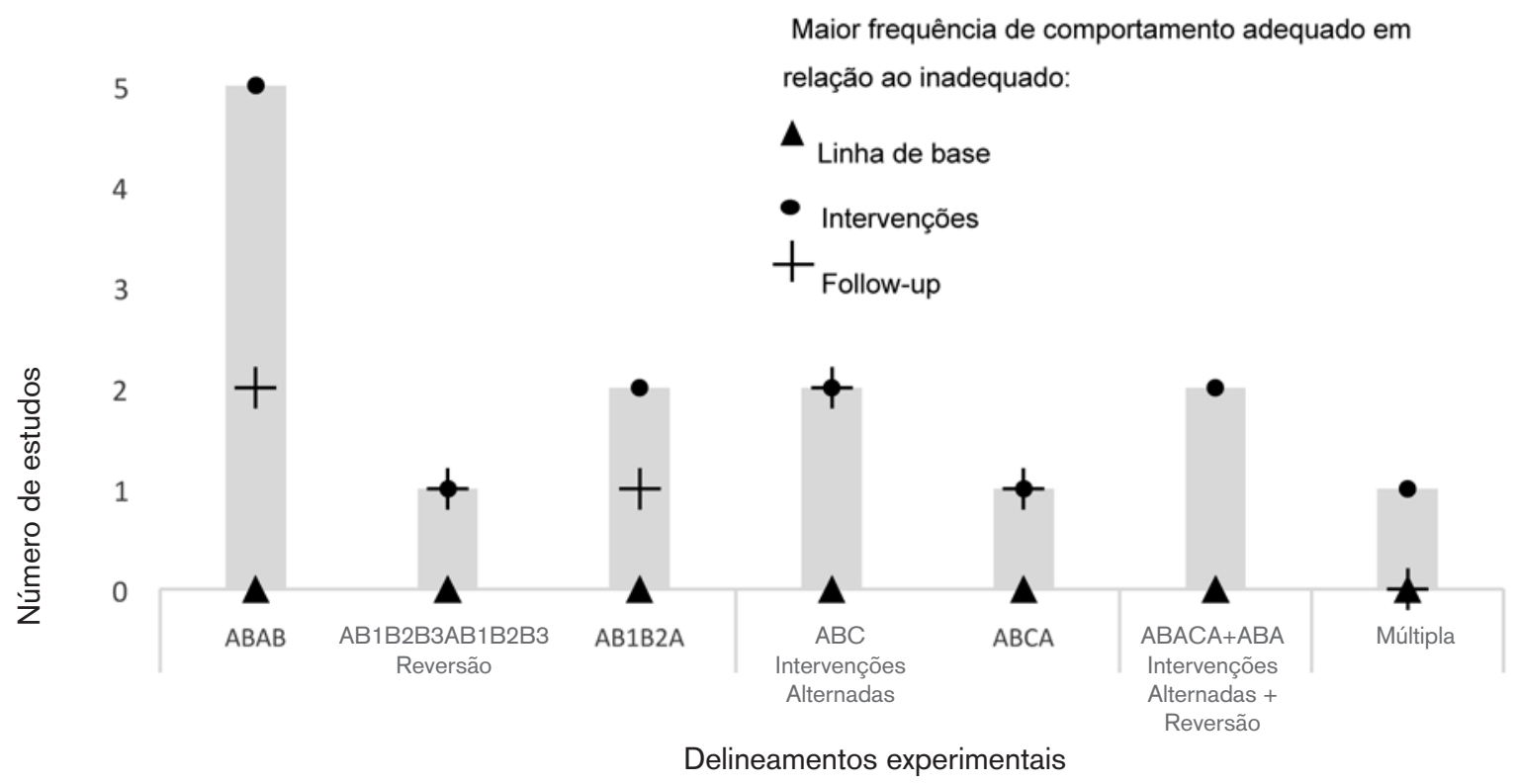

Figura 4. Delineamentos experimentais utilizados nos estudos. Os marcadores mostram o número de estudos nos quais a frequência do comportamento adequado foi maior que 0 inadequado nas fases de Linha de base (triângulo), intervernções (círculo) e follow-up (cruz). 
Vários foram os delineamentos experimentais empregados nas intervenções dos estudos. A Figura 4 sumariza esses delineamentos utilizados e os principais resultados obtidos, dado que os detalhes serão discutidos adiante. Os marcadores representam a quantidade de estudos nos quais o comportamento adequado (e.g.: falas adequadas, lavar as mãos, escovar os dentes, ler, beber água usando copo, etc.) foi registrado em maior frequência em relação ao inadequado nas fases de linha de base (triângulo), intervenções (círculo) e follow-up (cruz).

É possível observar que vários foram os delineamentos empregados nas intervenções dos estudos. O delineamento de reversão $\mathrm{ABAB}$ foi o mais utilizado, em cinco estudos, seguido pelo delineamento AB1B2A (2), o de intervenções alternadas $\mathrm{ABC}$ (2) e o delineamento de intervenções alternadas seguido por um delineamento de reversão $\mathrm{ABA}$ (2). Os delineamentos de reversão com vários tratamentos $\mathrm{AB} 1 \mathrm{~B} 2 \mathrm{~B} 3 \mathrm{AB} 1 \mathrm{~B} 2 \mathrm{~B} 3$, intervenções alternadas $A B C A$ e o de linha de base múltipla foram utilizados uma vez.

Embora cada estudo tenha objetivos e comportamentos-alvo distintos, foi possível indicar a quantidade de estudos nos quais a frequência do comportamento adequado foi maior que o inadequado a depender do delineamento utilizado.

Nota-se na Figura 4 que o comportamento adequado não foi mais frequente que o inadequado na linha de base em nenhum delineamento. Por outro lado, em todas as intervenções e delineamentos empregados, o comportamento adequado foi maior que o inadequado, mantendo-se no follow-up em todos os estudos que utilizaram o delineamento de reversão com múltiplos tratamentos e o de intervenções alternadas. Importante ressaltar que os estudos que utilizaram o delineamento de intervenções alternadas seguido por delineamento de reversão não tiveram follow-up.

A partir deste ponto do artigo pretende-se analisar as diferentes intervenções que foram planejadas nos estudos provenientes das buscas. As intervenções que foram realizadas e tiveram como foco a redução na frequência de falas inadequadas e o aumento nas falas adequadas mostraram que por meio do procedimento de extinção para falas inadequadas e reforçamento diferencial de respostas alternativas (DRA) para qualquer outro tipo de resposta verbal vocal é possível alcançar esse objetivo (Alves, 2018; Britto, Rodrigues, Santos \& Ribeiro, 2006; Bueno, 2012; Rockenbach, 2014; Santana, 2008).

Britto et al. (2006) ao utilizarem um delineamento de reversão $\mathrm{ABAB}$ com DRA para respostas verbais adequadas (e.g., atenção social vocal, balançar a cabeça, sorrir) e extinção para inadequadas (e.g., olhar para algo distante, se afastar do participante, não fazer nenhum comentário) encontraram menor frequência de falas inadequadas e maior frequência de apropriadas nas fases de intervenção e follow-up em relação às linhas de base. Embora as falas adequadas tenham sido maiores nas fases de intervenção do que no follow-up, a frequência emitida nesta última fase foi maior que nas de linha de base.

Em intervenção semelhante, Santana (2008) modificou apenas o procedimento de extinção, abrindo um livro e simulando leitura silenciosa diante das falas inadequadas. Falas adequadas foram encontradas em maior frequência nas intervenções e inadequadas em maiores frequências nas linhas de base e follow-up.

Um estudo com tratamentos diferenciados (Bueno, 2012) continha duas fases de linha de base e três de intervenções diferenciadas no delineamento de reversão (A-B1-B2-B3-A-B1-B2-B3). Na primeira intervenção a pesquisadora aplicava os procedimentos. Na segunda, pesquisadora e enfermeira e na terceira apenas a enfermeira. Os dados de um participante mostraram maior frequência de falas apropriadas nas fases de intervenções e, para o segundo participante, nas intervenções e no follow-up. Intervenção semelhante foi conduzida por Rockenbach (2014), na qual experimentador ou assistente social realizava os procedimentos ao utilizarem delineamento de reversão AB1B2A. Falas inapropriadas foram menores que falas apropriadas nas fases de intervenção e follow-up, respectivamente.

Em um delineamento de tratamentos alternados ABC, Alves (2018), na primeira intervenção ensinou os participantes a discriminar verbalmente os efeitos de falas inadequadas em si e em outras pessoas. Já na segunda intervenção DRA com extinção foram empregados, em que diante falas inadequadas o pesquisador não disponibilizava atenção social e olhava em direção aos objetos da sala. Em contrapartida, diante de falas adequadas, o pesquisador fazia contato visual, sorria e abordava 
o assunto em questão. Os resultados apontaram redução de falas inadequadas e aumento na frequência de falas apropriadas em ambas as intervenções, sem muitas diferenças, mantendo-se no follow-up para os três participantes.

Outros pesquisadores (Felipe, 2009; Santos, 2007), também preocupados com comportamentos verbais vocais apropriados e inapropriados, acrescentaram outras intervenções em suas pesquisas. Santos (2007), em um delineamento de intervenções alternadas com três fases de intervenção (ABACA), por exemplo, aplicou o procedimento padrão DRA e extinção na primeira intervenção. $\mathrm{Na}$ segunda, quando participante emitia falas inadequadas era questionada a relação funcional entre os elementos da fala e reforço social para falas adequadas continuou a ser aplicado contingente às falas adequadas. Ainda, um treino de habilidades sociais foi realizado posteriormente em um delineamento de reversão $\mathrm{ABA}$, em que foram utilizados os procedimentos de ensaio comportamental, fornecimento de instruções, modelação e retroalimentação para as falas adequadas. Os resultados mostraram menores taxas de respostas verbais inapropriadas no treino de habilidades sociais (Intervenção 3), na aplicação dos procedimentos de DRA + Extinção (Intervenção 1) e quando questionadas as relações funcionais do que o participante dizia (Intervenção 2), respectivamente (Santos, 2007).

Felipe (2009) também utilizou o delineamento de intervenções alternadas (ABACA) seguido pelo delineamento de reversão $\mathrm{ABA}$, objetivando as duas primeiras intervenções em apresentar uma análise funcional dos elementos expostos verbalmente pelo participante e treino de habilidades sociais com o participante e membro da família (e.g., demonstração de que elocuções inadequadas não ocasionavam consequências reforçadoras, ensaio comportamental, treino de um familiar para consequenciar diferencialmente as classes de respostas verbais do participante), respectivamente. Na terceira intervenção do segundo delineamento foi aplicado o procedimento de DRA e extinção. Os dados do primeiro delineamento apontaram maior número de falas inadequadas apenas na primeira linha de base. Com a introdução da primeira intervenção até o fim do estudo houve maior número de falas adequadas, chegando a maiores números na aná- lise funcional dos elementos da fala (Intervenção 1), treino de habilidades sociais com o participante e treino com membro da família (Intervenção 2), respectivamente. Com a posterior introdução do segundo delineamento, as falas apropriadas se mantiveram em maior frequência do que falas inapropriadas nas três fases, chegando a maiores números nas últimas sessões de intervenção e na última linha de base.

O uso de diferentes tratamentos de forma alternada foi realizado também por Sousa (2013) e Nogueira (2016). Sousa (2013) utilizou o delineamento $\mathrm{ABCA}$, sendo que a primeira intervenção foi a análise funcional dos elementos contidos nas falas inapropriadas com o participante e a segunda o procedimento de DRA e extinção. Os resultados mostraram maior frequência de falas inadequadas apenas na primeira fase de linha de base e na primeira sessão de ambas as intervenções. Falas adequadas, por outro lado, foram registradas em maior número nas demais sessões das duas intervenções, posterior linha de base e follow-up. Nogueira (2016) utilizou o delineamento $\mathrm{ABC}$, sendo a primeira intervenção o procedimento DRA e extinção e a segunda o ensino da discriminação da natureza de estruturas dos estímulos no ambiente social do participante, com descrições físicas e apropriadas do que ele ouvia, falava, olhava ou sentia. Maiores frequências de falas inapropriadas foram registradas apenas na primeira linha de base, invertendo para menores frequências nas duas intervenções. Menores frequências de falas inapropriadas e maiores de apropriadas foram registradas no fim da segunda intervenção, mantendo-se no follow-up.

Os dados dos estudos que investigaram respostas verbais sugerem que utilizar os procedimentos de extinção para falas inapropriadas e DRA para apropriadas parecem ser eficazes tanto na aplicação realizada pelo pesquisador quanto de outros profissionais treinados, apontando que falas inapropriadas de pessoas com diagnóstico de esquizofrenia podem ser mantidas por atenção social (Alves, 2018; Britto et al., 2006; Bueno, 2012; Felipe, 2009; Nogueira, 2016; Rockenbach, 2014; Santana, 2008; Santos, 2007; Sousa, 2013). Tratamentos diferentes (e.g., treino de habilidades sociais com participante, familiares e análise funcional dos elementos contidos nas falas inapropriadas) também mostraram 
redução nessas falas (Felipe, 2009; Nogueira, 2016; Santos, 2007; Sousa, 2013).

Os demais estudos que resultaram das buscas não tiveram necessariamente como alvo comportamentos verbais, mas outras respostas como, por exemplo, respostas agressivas (Miranda, 2005; Miranda \& Britto, 2011; Silva, 2005), varrer o pátio, limpar a mesa, desenhar, falar sobre si, mandos para pamonha (Silva, 2005), lavar as mãos antes do lanche da tarde e escovar os dentes em seguida, leitura, conversas, fala inadequada (Martone, 2005), contato visual, volume baixo da voz e verbalizações curtas (Epaminondas, 2010), manter contato olho a olho, atividade em tempo livre, bater palmas e beber água no copo (Miranda, 2005; Miranda \& Britto, 2011).

Os pesquisadores geralmente utilizavam de instruções para que o participante emitisse a resposta desejada: "solte meu braço, por favor", "quero que você varra ali para mim" e "faça um desenho para mim" (Silva, 2005), ficar ao lado fornecendo dicas e mostrar através de gestos e palavras, entregar uma revista e pedir para ler (Martone, 2005), "olhe para mim para eu te entender melhor", "fale um pouco mais alto para eu te entender melhor" (Epaminondas, 2005), "olhe dentro dos meus olhos", "quero que se aproxime daquele grupo", "observe o que eles estão fazendo e permaneça junto a eles por dois minutos" ou fornecer um copo e pedir para o participante usá-lo para beber água (Miranda, 2005; Miranda \& Britto, 2011). Nesses estudos, aprovação social foi utilizada como consequência para respostas desejáveis. Ainda, há estudos que utilizaram também comestíveis (Miranda, 2005; Miranda \& Britto, 2011; Silva, 2005). O procedimento de extinção foi utilizado para respostas agressivas (Miranda, 2005; Miranda \& Britto, 2011; Silva, 2005) ou para falas indaquadas (Martone, 2005).

Ao utilizarem o delineamento de reversão ABAB com follow-up (Miranda, 2005; Miranda \& Britto, 2011; Silva, 2005), linha de base múltipla (Epaminondas, 2010) ou treino de cadeias de respostas com cinco fases (Martone, 2005), os resultados dos estudos apontaram aumento na frequência de respostas desejáveis e diminuição em indesejáveis nas intervenções, em comparação com a linha de base (Epaminondas, 2010; Miranda, 2005; Miranda \& Britto, 2011; Silva, 2005), mantendo-se no follow-up (Silva, 2005). No estudo de Miranda (2005) e Miranda e Britto (2011) apenas as respostas agressivas mantiveram em baixa frequência no follow-up. Já no estudo de Martone (2005), respostas de higiene pessoal não tiveram suas frequências aumentadas na última fase (remoção de dicas e instruções). Porém, outras respostas de higiene, que não treinadas, foram emitidas durante as quatro primeiras fases da intervenção.

As pesquisas que tiveram como foco uma variedade de respostas e não apenas o comportamento verbal vocal dos participantes mostraram que é possível aumentar a frequência de respostas adaptativas e diminuir a de respostas indesejáveis por meio de procedimentos de reforçamento, extinção, modelação e o uso de instruções. Os reforçadores comumente utilizados foram atenção social e comestíveis. Ainda, esses estudos mostram a importância de planejar uma intervenção focada nos comportamentos do participante da pesquisa em seu ambiente natural, visto que a seleção de respostas-alvo parece corresponder às necessidades do participante da pesquisa, característica proveniente de pesquisas aplicadas (Baer et al., 1968).

\section{Conclusão}

As buscas realizadas em bases de dados apontaram um número menor de artigos publicados dentro dessa linha de pesquisa no Brasil em comparação com teses e dissertações realizadas, em sua maioria, na Pontifícia Universidade Católica de Goiás. Desde a década de 60 estudos foram conduzidos no exterior. No Brasil foi encontrado o primeiro publicado em 2005. Todavia, a literatura internacional apontou um decréscimo nas publicações nas últimas décadas enquanto que no Brasil parece haver produções constantes de teses e dissertações.

Embora o procedimento mais utilizado em intervenções para diminuição de respostas verbais inapropriadas (DRA e extinção) tenha mostrado eficácia, outros procedimentos apareceram nos estudos e também mostraram suas relevâncias como, por exemplo, o treino de habilidades sociais para o participante e familiar, modelação e análise funcional junto ao participante de elementos contidos em falas. 
Alguns dos estudos que trabalharam com respostas verbais vocais realizaram análise funcional experimental antes da implementação de intervenções, o que aponta uma preocupação dos pesquisadores em relação a demonstrar as variáveis sobre as quais o comportamento-alvo esteve sob controle. As diversas condições utilizadas apontaram que essa classe de respostas pode ser mantida por contingências de reforçamento.

Em relação aos comportamentos-alvo que usualmente foram selecionados nos estudos, comportamento verbal vocal apropriado e inapropriado foi o que mais os pesquisadores trabalharam, dado este que condiz com a literatura internacional das duas últimas décadas. Outros estudos tiveram como variáveis dependentes classes de respostas agressivas, de higiene pessoal, habilidades sociais, leitura, desenhar e também mostraram sua relevância ao utilizarem procedimentos de reforçamento, extinção, treino de cadeia de respostas e uso de instruções.

Mais estudos precisam ser conduzidos para fortificar a eficácia dos procedimentos encontrados nos estudos com a população com diagnóstico de esquizofrenia. Sugere-se que respostas de autocuidado, bem como habilidades sociais sejam foco de análise e intervenção em próximos estudos, visto que as pesquisas aqui apresentadas mostraram a efetividade em se trabalhar essas classes de respostas, que parecem ser importantes quando pensamos em comportamentos socialmente relevantes.

Outra sugestão é envolver os familiares e profissionais nos procedimentos que são implementados com os participantes. Em alguns estudos aqui apresentados, por exemplo, os pesquisadores treinaram profissionais que tinham contato com o participante para aplicar o procedimento de DRA com extinção reportando eficácia. Em outros, o treino de habilidades sociais foi realizado junto aos familiares. Ao pensar em estender a aplicação para pessoas que estão em convívio com o participante pode facilitar também a generalização dos procedimentos e resultados para outros contextos.

\section{Referências}

Alves, J. C. (2018). Análise funcional e tratamento das respostas verbais bizarras de pessoas com diagnóstico de esquizofrenia (Tese de Doutorado). Pontifícia Universidade Católica de Goiás, Goiânia.

American Psychiatric Association. (2014). DSM-V - Manual diagnóstico e estatístico de transtornos mentais. Porto Alegre: Artmed. (Trabalho original publicado em 2013).

Ayllon, T., \& Azrin, N. H. (1965). The measurement and reinforcement of behavior of psychotics. Journal of The Experimental Analysis of Behavior, 8(6), 357-383. doi: 10.1901/ jeab.1965.8-357

Ayllon, T., \& Haughton, E. (1964). Modification of symptomatic verbal behaviour of mental patients. Behavior Research and Therapy, 2(2-4), 87-97. doi: 10.1016/0005-7967(64)90001-4

Baer, D. M., Wolf, M. M., \& Risley, T. R. (1968). Some current dimensions of applied behavior analysis. Journal of Applied Behavior Analysis, 1(1), 91-97. doi: 10.1901/jaba.1968.1-91

Banaco, R. A. (1999). Técnicas cognitivo-comportamentais e análise funcional. Em R. R. Kerbauy \& R. C. Wielenska (Orgs.), Sobre comportamento e cognição (Vol. 4, pp. 75-82). Santo André: ESETec.

Bennett, P. S., \& Maley, R. F. (1973). Modification of interactive behaviors in chronic mental patients. Journal of Applied Behavior Analysis, 6(4), 609-620. doi: 10.1901/jaba.1973.6-609

Britto, I. A. G. S. (2004). Sobre delírios e alucinações. Revista Brasileira de Terapia Comportamental e Cognitiva, 6(1), 61-71.

Britto, I. A. G. S. (2005). Esquizofrenia: desafios para a ciência do comportamento. Em: Guilhardi, H. J. \& Aguirre, N. C. (Orgs.), Sobre comportamento e cognição (Vol. 16, pp. 38-44). Santo André: ESETec.

Britto, I. A. G. S., Marcon, R. M. (2019). Estudos descritivos e experimentais em contextos aplicados: dados científicos e impacto prático. Estudos de Psicologia, 24(2), 204-214. doi: 10.22491/1678-4669.20190022

Britto, I. A. G. S, Rodrigues, M. C. A., Santos, D. C. O., \& Ribeiro, M. A. (2006). Reforçamento 
diferencial de comportamentos verbais alternativos de um esquizofrênico. Revista Brasileira de Terapia Comportamental e Cognitiva, 8(1), 73-84. doi: 10.31505/rbtcc.v8i1.20

Britto, I. A. G. S., Rodrigues, I. S., Alves, S. L., \& Quinta, T. L. S. S. (2010). Análise funcional de comportamentos verbais inapropriados de um esquizofrênico. Psicologia: Teoria e Pesquisa, 26(1), 139-144. doi: 10.1590/S010237722010000100016

Bueno, G. N. (2012). Efeitos das condições de atenção e demanda nas respostas verbais de esquizofrênicos (Tese de doutorado). Pontifícia Universidade Católica de Goiás, Goiânia.

DeLeon, I. G., Arnold, K. L., Rodriguez-Catter, V., \& Uy, M. L. (2003). Covariation between bizarre and nonbizarre speech as a function of the content of verbal attention. Journal of Applied Behavior Analysis, 36(1), 101-104. doi: 10.1901/ jaba.2003.36-101

Dixon, M. R., Benedict, H., \& Larson, T. (2001). Functional analysis and treatment of inappropriate verbal behavior. Journal of Applied Behavior Analysis, 34(3), 361-36 3. doi: 10.1901/ jaba.2001.34-361

Epaminondas, F. R. (2010). Modelagem de comportamento para controle da esquizofrenia (Dissertação de mestrado). Pontifícia Universidade Católica de Goiás, Goiânia.

Felipe, G. R. (2009). Efeitos das estratégias operantes em uma pessoa com o diagnóstico de esquizofrenia e família (Dissertação de mestrado). Pontifícia Universidade Católica de Goiás, Goiânia.

Glynn, S. M. (1990). Token economy approaches for psychiatric patients. Behavior Modification, 14(4), 383-407. doi: 10.1177/01454455900144002.

Guedes, M. L. (1993). Equívocos da Terapia Comportamental. Temas em Psicologia, 2(1), 81-85.

Holland, J. (1978). Behaviorism: Part of the problem or part of the solution? Journal of Applied Behavior Analysis, 11(1), 163-174. doi: 10.1901/ jaba.1978.11-163

Iwata, B. A., Dorsey, M. F., Slifer, K. J., Bauman, K. E., \& Richman, G. S. (1994). Toward a functional analysis of self-injury. Journal of Applied
Behavior Analysis, 27(2), 197-209. doi: 10.1901/ jaba.1994.27-197

Kazdin, A. E. (1978). History of Behavior Modification: Experimental Foundations of Contemporary Research. Baltimore: University Park Press.

Laying, T. V. J., \& Andronis, P. T. (1984). Toward a functional analysis of delusional speech and hallucinatory behavior. The Behavior Analyst, 7(2), 139-156.

Liberman, R. P., Teigen, J., Patterson, R., \& Baker, V. (1973). Reducing delusional speech in chronic, paranoid schizophrenics. Journal of Applied Behavior Analysis, 6(1), 57-64. doi: 10.1901/ jaba.1973.6-57

Mace, F. C., \& Lalli, J. S. (1991). Linking descriptive and experimental analyses in the treatment of bizarre speech. Journal of Applied Behavior Analysis, 24(3), 553-562. doi: 10.1901/ jaba.1991.24-553

Marcon, R. M. (2010). O comportamento verbal do esquizofrênico sob múltiplas condições de controle (Dissertação de mestrado). Pontifícia Universidade Católica de Goiás, Goiânia.

Marcon, R. M. (2014). O controle pelos antecedentes e consequentes nas respostas verbais de pessoas com diagnóstico de esquizofrenia (Tese de doutorado). Pontifícia Universidade Católica de Goiás, Goiânia.

Marcon, R. M. \& Britto, I. A. G. S. (2015). Análise funcional de falas inapropriadas em uma pessoa com diagnóstico de esquizofrenia. Revista Brasileira de Análise do Comportamento, 11(1), 53-60. doi: 10.18542/rebac.v11i1.3774

Martone, R. C., \& Zamignani, D. R. (2002). Esquizofrenia: A análise do comportamento tem o que a dizer? Em Guilhardi, H. J., Madi, M. B. B. P., Queiroz, P. P., \& Scoz, M. C. (Orgs.), Sobre comportamento e Cognição (Vol. 10, pp. 305-316). Santo André: ESETec.

Martone, M. C. C. (2005). Efeitos de contingências discriminativas sobre os repertórios social e de higiene de uma participante institucionalizada (Dissertação de mestrado). Pontifícia Universidade Católica de São Paulo, São Paulo. Miranda, E. (2005). A esquizofrenia sob a perspectiva dos princípios da análise do comportamento (Dissertação de mestrado). Pontifícia 
Universidade Católica de Goiás, Goiânia.

Miranda, E., \& Britto, I. A. G. S. (2011). Aplicação dos princípios analítico-comportamentais para alterar o comportamento de uma esquizofrênica. Psicologia: Teoria e Pesquisa, 27(3), 327-336.

Moura, L. F. (2012). Esquizofrenia: estudo de falas inapropriadas sob múltiplas condições de controle (Dissertação de mestrado). Pontifícia Universidade Católica de Goiás, Goiânia.

Nelson, G. L., \& Cone, J. D. (1979). Multiplebaseline analysis of a token economy for psychiatric inpatients. Journal of Applied Behavior Analysis, 12(2), 255-271. doi: 10.1901/ jaba.1979.12-255

Nogueira, G. R. (2016). Avaliação e tratamento das falas inapropriadas de um indivíduo com o diagnóstico de esquizofrenia (Dissertação de mestrado). Pontifícia Universidade Católica de Goiás, Goiânia.

Rockenbach, B. P. (2014). Análise funcional das respostas verbais de uma pessoa com o diagnóstico de esquizofrenia (Dissertação de mestrado). Pontifícia Universidade Católica de Goiás, Goiânia.

Santana, L. A. M. (2008). Comportamento verbal e esquizofrenia: estratégia operante de intervenção (Dissertação de mestrado). Pontifícia Universidade Católica de Goiás, Goiânia.

Santos, D. C. O. (2007). Análise da fala psicótica via estratégias operantes de intervenção (Dissertação de mestrado). Pontifícia Universidade Católica de Goiás, Goiânia.

Santos, F. A., Santos, K. L., \& Aureliano, L. F. G. (2013). Estudo do comportamento psicótico pela análise do comportamento: revisão das publicações no JEAB e JABA. Revista Perspectivas em Análise do Comportamento, 4(1), 51-68.

Schock, K., Clay, C., \& Cipani, E. (1998). Making sense of schizophrenic symptoms: delusional statements and behavior may be functional in purpose. Journal of Behavior Therapy and Experimental Psychiatry, 29(2), 131-141. doi: 10.1016/S0005-7916(98)00010-X

Scotti, J. R., McMorrow, M. J., \& Trawitzki, A. L. (1993). Behavioral treatment of chronic psychiatric disorders: publication trends and future directions. Behavior Therapy, 24(4), 527-550. doi: 10.1016/S0005-7894(05)80316-8

Silva, K. P. L. (2005). Análise aplicada e o comportamento diagnosticado como esquizofrênico (Dissertação de mestrado). Pontifícia Universidade Católica de Goiás, Goiânia.

Skinner, B. F. (1959). What is psychotic behavior? In Cumulative Record (pp. 202-219). New York: Appleton Century Crofts.

Sousa, N. R. (2013). Múltiplas condições de controle no comportamento de uma pessoa com diagnóstico de esquizofrenia em comunidade evangélica (Dissertação de mestrado). Pontifícia Universidade Católica de Goiás, Goiânia.

Tracey, D. A., Briddell, D. W., \& Wilson, G. T. (1974). Generalization of verbal conditioning to verbal and nonverbal behavior: group therapy with chronic psychiatric patients. Journal of Applied Behavior Analysis, 7(3), 391-402. doi: 10.1901/jaba.1974.7-391

Wilder, D. A., Masuda, A., O'Connor, C., \& Baham, M. (2001). Brief functional analysis and treatment of bizarre vocalizations in an adult with schizophrenia. Journal of Applied Behavior Analysis, 34(1), 65-68. doi: 10.1901/ jaba.2001.34-65

Wincze, J. P., Leitenberg, H., \& Agras, W. S. (1972). The effects of token reinforcement and feedback on the delusional verbal behavior of chronic paranoid schizophrenics. Journal of Applied Behavior Analysis, 5(3), 247-262. doi: 10.1901/ jaba.1972.5-247

Winkler, R. C. (1970). Management of chronic psychiatric patients by a token reinforcement system. Journal of Applied Behavior Analysis, 3(1), 47-55. doi: 10.1901/jaba.1970.3-47

\section{Informações do Artigo}

Histórico do artigo:

Submetido em: 20/01/2020

Primeira decisão editorial: 04/08/2020

Aceito em: 23/09/2020

Editor associado: Daniel Afonso Assaz 